\title{
Winding planar probabilities
}

\author{
Jolanta Misiewicz • Jacek Wesołowski
}

Received: 26 March 2010 / Published online: 14 December 2010

(C) The Author(s) 2010. This article is published with open access at Springerlink.com

\begin{abstract}
The transformation of the plane which winds it up around the origin $k$ times is called $k$-winding. We study invariance properties of probability measures under $k$-windings, in particular, relations with rotation invariance in the first part of the paper. Then winding versions of the Bernstein theorem on characterization of the product of normal distributions are obtained. Finally, it is shown that the second component of a 2-winding of iid variables does not identify distributions even of squares of the original variables. This fact is in a sharp contrast to the property of the first component, distribution of which does determine uniquely the distribution of iid variables.
\end{abstract}

Keywords Winding - Rotation invariance - Normal distribution - Generalized inverse Gaussian distribution · Identification of probability distribution

\section{Introduction}

Rotation and winding are transformations of the plane $\mathbb{R}^{2}$. Rotation relies on shifting the polar angle while winding on taking multiple of the polar angle. Therefore, it will be convenient to introduce polar coordinates $(\rho, \theta) \in(0, \infty) \times[0,1)$ by

$$
(x, y)=(\rho \cos (2 \pi \theta), \rho \sin (2 \pi \theta)), \quad(x, y) \in \mathbb{R}^{2} \backslash\{(0,0)\} .
$$

\footnotetext{
J. Misiewicz · J. Wesołowski ( $\square)$

Politechnika Warszawska, Warszawa, Poland

e-mail: wesolo@mini.pw.edu.pl

J. Misiewicz

e-mail: j.misiewicz@mini.pw.edu.pl
} 
More precisely, $\alpha$-rotation $\mathcal{R}_{\alpha}$ (i.e. rotation by the angle $\alpha \in[0,2 \pi)$ ) is defined by

$$
\mathcal{R}_{\alpha}(x, y)=(\rho \cos (2 \pi \theta+\alpha), \rho \sin (2 \pi \theta+\alpha)), \quad(x, y) \in \mathbb{R}^{2} \backslash\{(0,0)\} .
$$

Analogously, for $k$ being a positive integer, $k$-winding $\mathcal{W}_{k}$ (i.e. winding the plane $k$-times around the origin) is defined by

$$
\mathcal{W}_{k}(x, y)=(\rho \cos (2 \pi k \theta), \rho \sin (2 \pi k \theta)) \quad(x, y) \in \mathbb{R}^{2} \backslash\{(0,0)\} .
$$

Additionally, we define $\mathcal{R}_{\alpha}(0,0)=\mathcal{W}_{k}(0,0)=(0,0)$.

Consequently, in polar coordinates $\mathcal{R}_{\alpha}$ is defined through

$$
(\rho, \theta) \mapsto\left(\rho, \operatorname{fr}\left(\theta+\frac{\alpha}{2 \pi}\right)\right),
$$

where $\operatorname{fr}(x)=x-\lfloor x\rfloor$ is the fractional part of $x \in \mathbb{R}$.

Similarly, $k$-winding $\mathcal{W}_{k}$ is defined in polar coordinates by

$$
(\rho, \theta) \mapsto(\rho, \operatorname{fr}(k \theta)) .
$$

Let $(X, Y)$ be a random vector assuming values in $\mathbb{R}^{2}$ and having the distribution without atom at zero (it will be our standing assumption throughout the paper). We define its polar representation $(R, \Theta)$ through

$$
(X, Y)=(R \cos (2 \pi \Theta), R \sin (2 \pi \Theta)) .
$$

The distribution of $(X, Y)$ is invariant under $\alpha$-rotation, that is $(X, Y) \stackrel{d}{=} \mathcal{R}_{\alpha}(X, Y)$, iff

$$
(R, \Theta) \stackrel{d}{=}\left(R, \text { fr }\left(\Theta+\frac{\alpha}{2 \pi}\right)\right) .
$$

Denote by $\mathcal{N}(0,1)$ the standard normal distribution in $\mathbb{R}$. It is well known that the product measure $\mathcal{N}(0,1) \otimes \mathcal{N}(0,1)$ is invariant under any rotation. The same holds true for the uniform measure on the unit circle in $\mathbb{R}^{2}$.

Similarly, the distribution of $(X, Y)$ is invariant under $k$-winding $\mathcal{W}_{k}$, that is $(X, Y) \stackrel{d}{=} \mathcal{W}_{k}(X, Y)$ iff

$$
(R, \Theta) \stackrel{d}{=}(R, \operatorname{fr}(k \Theta)) .
$$

Note that if $(X, Y)$ is $\mathcal{W}_{k}$ invariant then: (1) its projection on the unit circle $(\cos 2 \pi \Theta, \sin 2 \pi \Theta)$ is also $\mathcal{W}_{k}$ invariant, (2) $(T X, T Y)$ is $\mathcal{W}_{k}$ invariant for any $T \geq 0$ such that $T$ and $(X, Y)$ are independent.

Relations between invariance under windings and under rotations are discussed in Sect. 2. In particular, it is immediate that invariance under rotations implies invariance under windings. Results in the converse direction are somewhat more subtle. 
The celebrated Bernstein characterization of the normal law was concerned with the rotation $\mathcal{R}_{\pi / 4}$. Namely, Bernstein (1941) proved, under some technical assumptions, that: if $X$ and $Y$ are independent, and also $X+Y$ and $X-Y$ are independent, then $X$ and $Y$ are iid (independent identically distributed) normal. This classical result has been a source of numerous investigations of related issues. In Sect. 3 we derive analogues of this characterization result under 2-winding, i.e. instead of shifting the polar angle by $\pi / 4$, we double it.

Note that 2-winding in terms of variables $(x, y)$ is defined by

$$
\mathcal{W}_{2}(x, y)=\left(\frac{x^{2}-y^{2}}{\sqrt{x^{2}+y^{2}}}, \frac{2 x y}{\sqrt{x^{2}+y^{2}}}\right), \quad(x, y) \in \mathbb{R}^{2}
$$

with $\mathcal{W}_{2}(0,0)=(0,0)$ by continuity.

This transformation was considered by Shepp (1964), who observed that the product measure $\mathcal{N}(0,1) \otimes \mathcal{N}(0,1)$ is invariant under $\mathcal{W}_{2}$. In terms of random variables (rv's) it says that if $X$ and $Y$ are independent standard normal random variables then also

$$
W=\frac{X^{2}-Y^{2}}{\sqrt{X^{2}+Y^{2}}} \quad \text { and } \quad Z=\frac{2 X Y}{\sqrt{X^{2}+Y^{2}}}
$$

are independent and both have the standard normal distribution.

It has to be mentioned that $Z$ alone was considered in the context of characterization or identifiability questions. In particular Bansal et al. (1999) (for related multivariate considerations see Arellano-Valle 2001) obtained the following result: if $X$ and $Y$ are iid rv's and $Z$ is standard normal, then $X$ and $Y$ are also standard normal. This result has been recently extended to identifiability of the distribution of $X$ by the distribution of $Z$ in Hamedani et al. (2007). Apparently, a similar result cannot hold for $W$ since it is defined in terms of squares of $X$ and $Y$ (thus Cor. 2.4 of Bansal et al. 1999 is not correct). Nevertheless, one may expect that the assumption of normality of $W$ may lead to Chi-square distribution with one degree of freedom for $X^{2}$ and $Y^{2}$. However, as it will be shown in Sect. 4, there exist other distributions for $X^{2}$ and $Y^{2}$ for which $W$ is normal. Related problems were studied for instance in Beer and Lukacs (1973) and Hamedani and Volkmer (2001).

\section{Invariance under windings and invariance under rotations}

In this section we investigate relations between rotationally invariant and winding invariant probability measures. It will be convenient to use the Fourier-Laplace transform of the form

$$
\phi(k, s)=\mathbb{E} e^{i 2 \pi k \Theta-s R}, \quad(k, s) \in \mathbb{Z} \times(0, \infty),
$$

which uniquely determines the joint law of $(R, \Theta)$ and thus of $(X, Y)$. 
Recall that rotational invariance of the distribution of $(X, Y)$, i.e. the fact that $(X, Y) \stackrel{d}{=} \mathcal{R}_{\alpha}(X, Y)$ for any $\alpha \in \mathbb{R}$ is equivalent to

$$
\left(e^{i k \alpha}-1\right) \phi(k, s)=0, \quad \forall(k, s) \in \mathbb{Z} \times(0, \infty),
$$

holding $\forall \alpha \in \mathbb{R}$, which is equivalent to

$$
\phi(k, s)=0 \quad \forall(k, s) \in \mathbb{Z} \times(0, \infty), k \neq 0 .
$$

Furthermore, it is equivalent to $c_{k}=\mathbb{E} e^{i 2 \pi k \Theta}=0, \forall k \in \mathbb{Z} \backslash\{0\}$ (meaning that $\Theta$ is uniform on $(0,1))$ together with independence of $\Theta$ and $R$.

Note that if $\alpha_{0}$ is a fixed real number such that $\frac{\alpha_{0}}{2 \pi}$ is irrational then $k \alpha_{0}$ is never a multiple of $2 \pi$. Consequently, it follows by (1) that invariance by $\mathcal{R}_{\alpha_{0}}$ is equivalent to rotational invariance.

Invariance by $k$-winding of the distribution of $(X, Y)$ in terms of $\phi$ can be equivalently stated as

$$
\phi(k l, s)=\phi(l, s) \quad \forall(l, s) \in \mathbb{N} \times(0, \infty), l \neq 0 .
$$

First, we observe that rotation invariance implies winding invariance.

Proposition 2.1 Assume that the distribution of $(X, Y)$ is rotationally invariant. Then it is invariant under $k$-winding $\mathcal{W}_{k}$ for any integer $k \geq 1$.

Proof By (2) it follows that (3) holds with both sides of the equation equal to zero.

Note that there exists $(W, Z)=\mathcal{W}_{2}(X, Y)$ which is rotationally invariant while $(X, Y)$ is not. To see this consider

$$
(X, Y)=(R \cos 2 \pi \Theta, R \sin 2 \pi \Theta)
$$

for $\Theta$ with uniform distribution on $\left[\theta_{0}, \frac{1}{2}+\theta_{0}\right)$ independent of the nonnegative random variable $R$. The distribution of $(X, Y)$ is then concentrated on a half-plane, but $(\cos 4 \pi \Theta, \sin 4 \pi \Theta)$ has uniform distribution on the unit circle $S_{1} \subset \mathbb{R}^{2}$ thus $(W, Z)$ has a rotationally invariant distribution.

The rest of this section is devoted to study the converse relations, i.e. to what extent invariance with respect to windings implies invariance with respect to rotations.

Theorem 2.2 Let $k>1$ be a fixed integer. If distribution of the random vector $(X, Y)$ is invariant under both the rotation $\mathcal{R}_{\frac{2 \pi}{k}}$ and the winding $\mathcal{W}_{k}$, then it is rotationally invariant.

Proof By (1) with $\alpha=\frac{2 \pi}{k}$ we have

$$
\left(e^{i 2 \pi \frac{l}{k}}-1\right) \phi(l, s)=0 \quad \forall(l, s) \in \mathbb{Z} \times(0, \infty) .
$$


Consequently, $\phi(l, s)=0$ for any $s \in(0, \infty)$ and any $l \in \mathbb{Z}$ which is not a multiple of $k$. If $l \neq 0$ is a multiple of $k$ then it can be decomposed as $l=k^{r} m$ where $r$ is a positive integer and $m$ is an integer which is not a multiple of $k$. Then by (3) we get

$$
\phi(l, s)=\phi(m, s)
$$

and thus, by what we have already established, $\phi(m, s)=0$. Finally, we conclude that (2) holds and thus $(X, Y)$ is rotationally invariant.

It appears that if a distribution is invariant under all possible windings then it is almost rotationally invariant.

Theorem 2.3 Let $(X, Y) \stackrel{d}{=} \mathcal{W}_{k}(X, Y)$ for any $k=1,2, \ldots$ Then

$$
(X, Y) \stackrel{d}{=} Z\left(X_{1}, 0\right)+(1-Z)\left(X_{2}, Y_{2}\right)
$$

where $Z, X_{1}$ and $\left(X_{2}, Y_{2}\right)$ are independent, $Z$ is Bernoulli, $X_{1}$ is positive and $\left(X_{2}, Y_{2}\right)$ is rotationally invariant.

Proof Note that the distribution of $\left(X_{1}, 0\right)$ is invariant under any winding. Therefore, without any loss of generality it suffices to show that if in the polar representation $(\Theta, R)$ of $(X, Y)$ the polar angle does not have an atom at zero, then $(X, Y)$ is rotationally invariant.

Returning to the Fourier-Laplace transform, we note that to prove the result it suffices to show that $\phi(k, s)=0$ if only $k \neq 0$ (see 2).

By the invariance assumption through (3) and taking conjugates we get

$$
\phi(k, s)=\phi(1, s)=\overline{\phi(-1, s)}=\overline{\phi(-k, s)}, \quad \forall k=1,2, \ldots, \forall s>0 .
$$

Now, for an arbitrary and fixed $s>0$ consider a new random variable $\widetilde{\Theta}_{s}$ on $[0,1)$ whose Fourier transform is $\phi(k, s) / \phi(0, s)$. To finish the proof it suffices to show that $\widetilde{\Theta}_{s}$ is uniform on $(0,1)$. In this way we reduced the proof to the problem which is solved below in Lemma 2.4.

Lemma 2.4 Assume that the random variable $\Xi$ in $[0,2 \pi)$ has no atom at zero and

$$
\mathbb{E} e^{i k \Xi}=\mathbb{E} e^{i \Xi}=c_{1}, \quad \forall k=1,2, \ldots
$$

Then $c_{1}=0$ and $\Xi$ is uniform.

Proof Note that

$$
\eta_{n}=\frac{1}{n} \sum_{k=1}^{n} e^{i k \Xi}=\frac{e^{i(n+1) \Xi}-1}{n\left(e^{i \Xi}-1\right)} \rightarrow 0, \quad \text { a.s. }
$$

On the other hand, by (4) we have

$$
\mathbb{E} \eta_{n}=\mathbb{E} e^{i \Xi}=c_{1} .
$$


Since $\left|\eta_{n}\right|<1$ then by the Lebesgue dominated convergence we get that $c_{1}=0$. Consequently $c_{k}=0$ for any $k= \pm 1, \pm 2, \ldots$ Hence, $\Xi$ is uniform on $[0,2 \pi]$.

Note, that alternatively, the result of Theorem 2.3 , can be formulated as: if $\mu$ is a probability measure on $\mathbb{R}^{2}$ which is invariant under $k$-winding for any $k=1,2, \ldots$, then it is a convex combination of some distribution concentrated on $(0, \infty) \times\{0\}$ and some rotationally invariant probability measure on $\mathbb{R}^{2}$.

One could expect that if a probability measure is invariant under $\mathcal{W}_{k}$ and $\mathcal{W}_{l}$ for $k$ and $l$ with the only common divisor equal one, then the measure is rotationally invariant or discrete uniform, when restricted to the unit circle. However this is not the case, as it is explained in the following example.

Example 1 Consider $\Theta$ with the following distribution

$$
\mathbb{P}(\Theta=i / 5)=1 / 8, \quad i=1, \ldots, 4, \quad \text { and } \mathbb{P}(\Theta=i / 7)=1 /(12), \quad i=1, \ldots, 6
$$

Then it can be easily checked that $(X, Y)=(R \cos 2 \pi \Theta, R \sin 2 \pi \Theta)$, with $R$ and $\Theta$ independent, is invariant under $\mathcal{W}_{2}$ and $\mathcal{W}_{3}$.

The proof of the following lemma is based on standard calculations and will be omitted.

Lemma 2.5 Let $(X, Y)=(R \cos 2 \pi \Theta, R \sin 2 \pi \Theta)$ be a random vector which is invariant under the winding $\mathcal{W}_{k}$ for a fixed integer $k>1$. Then for every Borel set $A \subset[0, \infty)$ and every $0<a<b<1$

$$
\mathbb{P}\{R \in A, a<\Theta<b\}=\sum_{m=0}^{k-1} \mathbb{P}\left\{R \in A, \frac{a}{k}<\Theta-\frac{m}{k}<\frac{b}{k}\right\}
$$

Note that if the measure $\mu$ on $\mathbb{R}^{2}$ is invariant under $k$-winding then its "discrete" part (concentrated on rays) is also invariant under $k$-winding. The following result shows that if there is an open angular region (with vertex in the origin) outside of the support of $\mu$ then this measure is concentrated in

$$
\{(\rho \cos 2 \pi \theta, \rho \sin 2 \pi \theta): \rho \geq 0, \theta \in \mathbb{Q}\},
$$

where $\mathbb{Q}$ denotes the set of rational numbers in $[0,1]$.

Proposition 2.6 Let $(X, Y)=(R \cos 2 \pi \Theta, R \sin 2 \pi \Theta)$ be a random vector which is invariant under the winding $\mathcal{W}_{k}$ for a fixed integer $k>1$. Assume that there exist $a, b \in(0,1), a<b$ such that $\mathbb{P}(a<\Theta<b)=0$. Then $\mathbb{P}(\Theta \in \mathbb{Q})=1$.

Proof Since $(X, Y)$ is invariant under $\mathcal{W}_{k}$ then it is invariant also under $\mathcal{W}_{k^{n}}$. Thus applying Lemma 2.5 with $A=[0, \infty)$ we obtain

$$
0=\mathbb{P}\{a<\Theta<b\}=\sum_{m=0}^{k^{n}-1} \mathbb{P}\left(\frac{a+m}{k^{n}}<\Theta<\frac{b+m}{k^{n}}\right)
$$


and consequently for any $n \in \mathbb{N}$ and any $m \leq k^{n}-1$

$$
\mathbb{P}\left(\frac{a+m}{k^{n}}<\Theta<\frac{b+m}{k^{n}}\right)=0 .
$$

The proof will be completed if we show that for every irrational number $x \in(0,1)$ there exists $n_{0} \in \mathbb{N}$ and $m_{0} \leq k^{n_{0}}-1$ such that

$$
x \in\left(\frac{a+m_{0}}{k^{n_{0}}}, \frac{b+m_{0}}{k^{n_{0}}}\right) .
$$

To see this note first that the set $\left\{\operatorname{fr}\left(k^{n} x\right): n \in \mathbb{N}\right\}$ is dense in $[0,1]$, so there exists $n_{0} \in \mathbb{N}$ such that $\operatorname{fr}\left(k^{n_{0}} x\right) \in(a, b)$. Now, it is enough to define $m_{0}=\left\lfloor k^{n_{0}} x\right\rfloor$ and use the relation $k^{n_{0}} x=\left\lfloor k^{n_{0}} x\right\rfloor+\operatorname{fr}\left(k^{n_{0}} x\right)$.

The reverse implication in Proposition 2 does not hold, as we show in the example below.

Example 2 Denote by $\Pi$ the set of prime numbers which are greater than 2 . For every $k \in \Pi$ let

$$
\Pi_{k}=\left\{\frac{i}{k}, i=1, \ldots, k-1\right\} .
$$

Let

$$
\mathbb{P}(\Theta=x)=\frac{p_{k}}{k-1}, \quad x \in \Pi_{k}, \quad k \in \Pi,
$$

with $p_{k}>0, k \in \Pi$,such that $\sum_{k \in \Pi} p_{k}=1$. Then $\Theta$ is concentrated on the set $\bigcup_{k \in \Pi} \Pi_{k}$, a subset of $\mathbb{Q}$ which is dense in $(0,1)$. Since $\operatorname{fr}\left(2 \Pi_{k}\right)=\Pi_{k}$ for any $k \in \Pi$, it is immediate to see that $(X, Y)=(R \cos 2 \pi \Theta, R \sin 2 \pi \Theta)$, with $R$ and $\Theta$ independent, is $\mathcal{W}_{2}$ invariant.

On the other hand, smoothness assumption can be helpful.

Proposition 2.7 Let $(X, Y)=(R \cos 2 \pi \Theta, R \sin 2 \pi \Theta)$ be $\mathcal{W}_{k}$-invariant for an arbitrary fixed $k \geq 2$. If the distribution of $(X, Y)$ is absolutely continuous then it is rotationally invariant.

Proof Since $(X, Y)$ is absolutely continuous then also $(R, \Theta)$ is absolutely continuous. Therefore for any $s \geq 0$

$$
\lim _{n \rightarrow \infty} \phi(n, s)=0
$$

Iterating (3) we get

$$
\phi(l, s)=\phi\left(k^{n} l, s\right)
$$

for any $l \neq 0$ and any $n \in \mathbb{N}$. Now, (6) implies $\phi(l, s)=0$ for any $l \neq 0$ and any $s \geq 0$. That is, we obtained (2). Consequently, $(X, Y)$ is rotationally invariant. 


\section{Bernstein-like characterizations under winding}

The Shepp property implies that for standard normal variables winding preserves independence. The main objective of this section is to study converses of this observation. First we obtain the Bernstein-like characterization with rotation by $\frac{\pi}{4}$ changed into 2-winding under additional smoothness assumptions.

Theorem 3.1 Assume that $X, Y$ are symmetric, independent random variables having strictly positive continuous densities on $\mathbb{R}$. Let $(W, Z)=\mathcal{W}_{2}(X, Y)$. Assume that $W$ is symmetric. If $W$ and $Z$ are independent then $X$ and $Y$ are iid zero mean normal variables.

Proof Define random variables $R$ and $\Theta$ by $X=R \cos 2 \pi \Theta$ and $Y=R \sin 2 \pi \Theta$. Then $W=R \cos 4 \pi \Theta$ and $Z=R \sin 4 \pi \Theta$. Denote by $f_{i}, i=1,2,3$, 4, respectively, the densities of $X, Y, W$ and $Z$. Note that

$$
f_{R, \Theta}(r, \theta)=2 \pi r f_{1}(r \cos 2 \pi \theta) f_{2}(r \sin 2 \pi \theta)
$$

and for $\Xi=\operatorname{fr}(2 \Theta)$ we have

$$
f_{R, \Xi}(r, \xi)=2 \pi r f_{3}(r \cos 2 \pi \xi) f_{4}(\sin 2 \pi \xi)
$$

Since

$$
f_{R, \Xi}(r, \xi)=\frac{1}{2}\left(f_{R, \Theta}\left(r, \frac{\xi}{2}\right)+f_{R, \Theta}\left(r, \frac{\xi+1}{2}\right)\right)
$$

for almost all $r>0$ and $\xi \in(0,1)$ we arrive at

$2 f_{3}(r \cos 2 \pi \xi) f_{4}(\sin 2 \pi \xi)=f_{1}(r \cos \pi \xi) f_{2}(r \sin \pi \xi)+f_{1}(-r \cos \pi \xi) f_{2}(-r \sin \pi \xi)$

Since $X$ and $Y$ are symmetric the densities $f_{1}$ and $f_{2}$ are even functions and thus

$$
f_{1}(r \cos \pi \xi) f_{2}(r \sin \pi \xi)=f_{3}(r \cos 2 \pi \xi) f_{4}(r \sin 2 \pi \xi)
$$

holds for almost all $r>0$ and $\xi \in(0,2)$. Due to continuity of $f_{1}$ and $f_{2}$ the above equation holds for any $(r, \xi) \in(0, \infty) \times[0,2]$. Substituting $\pi \xi=$ : $\theta$ we arrive at

$$
f_{1}(r \cos \theta) f_{2}(r \sin \theta)=f_{3}(r \cos 2 \theta) f_{4}(r \sin 2 \theta)
$$

holding for any $\theta \in[0,2 \pi]$.

Plugging $\theta=0$ into (7) we get $f_{1}(r) f_{2}(0)=f_{3}(r) f_{4}(0)$ for any $r \geq 0$. By symmetry assumption $f_{3}$ is also an even function and thus

$$
f_{1}(r) f_{2}(0)=f_{3}(r) f_{4}(0)
$$


holds for any $r \in \mathbb{R}$. Integrating both sides of the above equation over $\mathbb{R}$ we get $f_{2}(0)=$ $f_{4}(0)$ and thus $f_{1}=f_{3}$. Plugging $\theta=\pi / 2$ into (7) we obtain that $f_{1}(0) f_{2}(r)=$ $f_{3}(-r) f_{4}(0)$, for any $r \geq 0$. Similarly as above we obtain $f_{2}=f_{3}$. Now, $\theta=\pi / 3$ in (7) leads to $f_{2}(r)=f_{4}(r)$ for any $r \geq 0$ and $\theta=2 \pi / 3$ gives $f_{2}(r)=f_{4}(-r), r \geq 0$. Therefore $f_{4}$ is an even function and thus $f_{2}=f_{4}$. Summing up, $f_{i}=f, i=1,2,3,4$, where $f$ is an even function.

By assumption $f$ is strictly positive, thus $G=\log f$ is well defined. Rewriting (7) in terms of $G$ gives

$$
G(r \cos \theta)+G(r \sin \theta)=G(r \cos 2 \theta)+G(r \sin 2 \theta),
$$

for any $r \geq 0$ and $\theta \in[0,2 \pi]$. Substituting $\theta=\alpha / 2$ for $\alpha \in(0, \pi / 2)$ in the Eq. (8) we obtain after $n$ iterations

$$
G(r \cos \alpha)+G(r \sin \alpha)=G\left(r \cos \frac{\alpha}{2^{n}}\right)+G\left(r \sin \frac{\alpha}{2^{n}}\right) .
$$

Since $G$ is a continuous function then letting $n \rightarrow \infty$ we obtain that for every $a, b \geq 0$

$$
G(a)+G(b)=G\left(\sqrt{a^{2}+b^{2}}\right)+G(0) .
$$

Define $H(a):=G(\sqrt{a})-G(0), a \geq 0$. Then we have

$$
\forall a, b>0 \quad H(a)+H(b)=H(a+b) .
$$

This is a classical Cauchy functional equation with the solution $H(t)=C t$, thus consequently $G(t)=C t^{2}+D$ for any $t \in \mathbb{R}$ since $G$ is even. Thus $f(t)=\exp \left(C t^{2}+D\right)$. It means that all the variables $X$ and $Y$ have zero mean normal distributions.

Corollary 3.2 Let $\mu$ be a symmetric probability measure on $\mathbb{R}^{2}$ having strictly positive continuous density. If the rotation $\mathcal{R}_{\pi / 4}$ and the winding $\mathcal{W}_{2}$ both transform $\mu$ into product measures, then $\mu$ is a product of identical normal, zero mean measures.

Proof Let $(X, Y)$ be a random vector with the distribution $\mu$. Then it follows that

$$
U=\frac{X+Y}{\sqrt{2}} \quad \text { and } \quad V=\frac{X-Y}{\sqrt{2}}
$$

are independent, and also

$$
W=\frac{X^{2}-Y^{2}}{\sqrt{X^{2}+Y^{2}}} \quad \text { and } \quad Z=\frac{2 X Y}{\sqrt{X^{2}+Y^{2}}}
$$

are independent. Note that

$$
W=\frac{2 U V}{\sqrt{U^{2}+V^{2}}} \quad \text { and } \quad Z=\frac{U^{2}-V^{2}}{\sqrt{U^{2}+V^{2}}} .
$$


Since $U, V$ are symmetric random variables with strictly positive continuous densities it follows from Theorem 3.1 that they have normal $N\left(0, \sigma^{2}\right)$ distributions, thus also $X$ and $Y$ are independent normal $N\left(0, \sigma^{2}\right)$.

If the original random variables $X$ and $Y$ in Theorem 3.1 are assumed to be not only independent, but also iid then the other assumptions may be considerably relaxed.

Proposition 3.3 Assume that $X$ and $Y$ are symmetric iid rv's and $(W, Z)=$ $\mathcal{W}_{2}(X, Y)$. If $W$ and $Z$ are independent, then $X$ and $Y$ have identical zero mean normal distribution.

Proof Denote $U=X^{-2}$ and $V=Y^{-2}$. Then $U$ and $V$ are positive non-degenerate iid rv's. Also

$$
W^{2}=\frac{1}{U}+\frac{1}{V}-\frac{4}{U+V} \quad \text { and } \quad Z^{-2}=U+V
$$

are independent. This condition is known to characterize the inverse Gaussian distribution (see Letac and Seshadri 1985), i.e. $U$ and $V$ are inverse Gaussian. Consequently, $X$ and $Y$ have continuous densities. Now the result follows from Theorem 3.1.

Note that the assumption of independence of $W^{2}$ and $Z^{2}$ (instead of independence of $W$ and $Z$ ) by the argument given in the proof above leads to characterization of $X$ to have the unique symmetric distribution such that $X^{-2}$ has an inverse Gaussian distribution.

The independence condition in this case can be even weakened to the constant regression assumption, i.e.

$$
\mathbb{E}\left(W^{2} \mid Z^{2}\right)=\text { const. }
$$

This is due to the fact that the above condition in terms of $U$ and $V$ introduced in the proof above resolves to

$$
\mathbb{E}\left(\frac{1}{U}+\frac{1}{V}-\frac{4}{U+V} \mid U+V\right)=\text { const. }
$$

It is known, see for instance Seshadri (1983), where additional moments assumptions are needed (but the r.v's are not necessarily positive), that the above condition implies that $U$ and $V$ are inverse Gaussian. For positive variables, the proof of this fact follows along the line of the argument given in the case of the independence assumption in Letac and Seshadri (1985).

\section{Does the distribution of $W$ determines $X$ and $Y$ ?}

Recall that $W$ was defined as the first component of $\mathcal{W}_{2}(X, Y)$, i.e.

$$
W=\frac{X^{2}-Y^{2}}{\sqrt{X^{2}+Y^{2}}} .
$$


Moreover, its square can be represented as

$$
W^{2}=\frac{1}{U}+\frac{1}{V}-\frac{4}{U+V},
$$

where $U=X^{-2}$ and $V=Y^{-2}$.

Shepp (1964) observed that if $X$ and $Y$ are independent normal $\mathcal{N}(0,1)$ then $W$ is also normal $\mathcal{N}(0,1)$. Obviously, the distribution of squares $X^{2}$ and $Y^{2}$ matters only. In this section we study the question if the chi-square distribution with one degree of freedom, $\chi^{2}(1)$, is the only common distribution of $X^{2}$ and $Y^{2}$ for which $W$ is standard normal. As it has already been mentioned in the Introduction, similar questions for the second element of the Shepp $\mathcal{W}_{2}$ transformation led to characterizations of the standard normal distribution (see Bansal et al. 1999) and even to more general identifiablity results (see Hamedani et al. 2007). In the case of $W$ the situation is drastically different.

Recall that the generalized inverse Gaussian distribution $G I G(p, a, b)$ is defined by the density

$$
f(x) \propto x^{p-1} \exp \left(-a x-\frac{b}{x}\right), \quad x \in \mathbb{R},
$$

where $p \in \mathbb{R}$ and $a, b \geq 0(a>0$ if $p \geq 0$ and $b>0$ if $p \leq 0)$ are the parameters (see for instance Jorgensen 1982). The normalizing constant is expressed in terms of the modified Bessel function of the third type and we skip it since its form is not important for our considerations. The GIG distribution has the following inversion property, i.e. $A \sim G I G(p, a, b)$ if and only if $A^{-1} \sim G I G(-p, b, a)$. An important particular case of GIG distributions is the inverse Gaussian distribution, IG, which occurs if the parameter $p=-\frac{1}{2}$, i.e. $I G(a, b)=G I G\left(-\frac{1}{2}, a, b\right), a \geq 0, b>0$. For more information on IG distributions we recommend the monograph by Seshadri (1993). Note that if a random variable $A$ has the $\chi^{2}(1)$ distribution then its inverse $A^{-1}$ has the inverse Gaussian distribution $I G\left(0, \frac{1}{2}\right)$ the only positive stable distribution with a closed analytical form of the density.

Proposition 4.1 Let $X, Y$ be independent random variables such that $X^{2}$ and $Y^{2}$ have the same distribution $G I G\left(\frac{1}{2}, \frac{1}{2}, a\right)$ for an arbitrary $a \geq 0$. Then $W$ (defined in (9)) is standard normal.

Proof Note that (9) implies that the distribution of $W$ is symmetric if only $X^{2}$ and $Y^{2}$ are iid. Therefore its suffices to show that $W^{2}$ has the $\chi^{2}(1)$ distribution.

Now we refer to the representation (10), that is we define $U=X^{-2}$ and $V=$ $Y^{-2}$ and $S=W^{2}$. By the property of the GIG distribution it follows that $U, V \sim$ $G I G\left(-\frac{1}{2}, a, \frac{1}{2}\right)$, that is they have a common density $f$ of the form

$$
f(x) \propto x^{-\frac{3}{2}} e^{-a x-\frac{1}{2 x}} I_{(0, \infty)}(x)
$$


Additionally, we define $T=U+V$. Note that the transformation

$$
\psi(u, v)=\left(\frac{1}{u}+\frac{1}{v}-\frac{4}{u+v}, u+v\right)
$$

is a bijection between $\{(u, v): 0<u<v\}$ onto $(0, \infty)^{2}$ with the inverse

$$
\psi^{-1}(s, t)=\left(\frac{t(1-r(s t))}{2}, \frac{t(1+r(s t))}{2}\right)
$$

where $r(x)=\sqrt{\frac{x}{4+x}}$. The map $\psi$ is also a bijection between $\{(u, v): 0<v<u\}$ onto $(0, \infty)$ with the inverse

$$
\psi^{-1}(s, t)=\left(\frac{t(1+r(s t))}{2}, \frac{t(1-r(s t))}{2}\right) .
$$

Therefore, after a lengthy but standard process of computing Jacobians in both cases (they differ only by sign), we arrive at

$$
f_{(S, T)}(s, t)=\frac{u^{2} v^{2}}{\left|v^{2}-u^{2}\right|} f\left(\frac{t(1-r(s t))}{2}\right) f\left(\frac{t(1+r(s t))}{2}\right),
$$

where $f$ is the common density of $U$ and $V$.

Consequently, we get

$$
\begin{aligned}
& f_{(S, T)}(s, t) \propto \frac{\frac{t^{4}}{16}(1+r(s t))^{2}(1-r(s t))^{2}}{\frac{t^{2}}{4} r(s t)}\left(\frac{t^{2}}{4}\left(1-r^{2}(s t)\right)\right)^{-\frac{3}{2}} \\
& e^{-a t-\frac{1}{t(1-r(s t))}-\frac{1}{t(1+r(s t))}} I_{(0, \infty)^{2}}(s, t) .
\end{aligned}
$$

This expression simplifies to

$$
f_{(S, T)}(s, t) \propto s^{-\frac{1}{2}} e^{-\frac{s}{2}} I_{(0, \infty)}(s) t^{-\frac{3}{2}} e^{-a t-\frac{2}{t}} I_{(0, \infty)}(t),
$$

meaning that $S$ and $T$ are independent and $S$ has the chi-square $\chi(1)$ distribution ( $T$ is an inverse Gaussian variable).

Note that the family of $G I G\left(\frac{1}{2}, \frac{1}{2}, a\right), a \geq 0$, contains the $\chi^{2}(1)$ distribution which occurs for $a=0$. Note also that it is the family of conditional distributions of $Y$ given $Z Y=a, a \geq 0$, where $Z$ and $Y$ are independent, $Z$ is exponential with the mean 1 , and $Y$ is gamma with the shape $3 / 2$ and the scale $1 / 2$. Related questions have been recently studied in Arnold and Seshadri (2009) and in matrix-variate setting in Seshadri and Wesołowski (2008). Moreover, if $X^{2} \sim G I G\left(\frac{1}{2}, \frac{1}{2}, a\right)$ and $X$ is symmetric then the 
density of $X$ is

$$
f(x) \propto \exp \left(-\frac{x^{2}}{2}-\frac{a}{x^{2}}\right),
$$

which can be considered as generalized normal density parameterized by $a \geq 0$.

It would be interesting to know if the family of probability laws $\left\{G I G\left(\frac{1}{2}\right.\right.$, $\left.\left.\frac{1}{2}, a\right), a \geq 0\right\}$ covers all possible distributions for $X^{2}$ and $Y^{2}$ which imply that $W$ has the standard normal distribution. This question remains open.

Acknowledgments We are deeply indebted to Gérard Letac whose comments led to improvements in Sect. 2 and to V. Seshadri for discussions regarding Proposition 4.1. Thanks are also due to referees for valuable remarks. In particular, comments on proofs of Theorem 3.1 and Proposition 4.1 are much appreciated.

Open Access This article is distributed under the terms of the Creative Commons Attribution Noncommercial License which permits any noncommercial use, distribution, and reproduction in any medium, provided the original author(s) and source are credited.

\section{References}

Arellano-Valle RB (2001) On some characterizations of spherical distributions Statist. Probab Lett 54:227232

Arnold B, Seshadri V (2009) Some independence properties of the inverse Gaussian law. Sankhya 71(A(1)):94-108

Bansal N, Hamedani GG, Key E, Volkmer H, Zhang H, Behboodian J (1999) Some characterizations of the normal distribution. Stat Probab Lett 42:393-400

Beer S, Lukacs E (1973) Characterizations of the normal distribution by suitable transformations. J Appl Probab 10:100-108

Bernstein SN (1941) On a characteristic property of the normal law. Trudy Leningr Polytekh Inst 3:21-22 (in Russian)

Hamedani GG, Volkmer H (2001) Certain characterizations of normal distribution via transformations. J Multivar Anal 77:286-294

Hamedani GG, Volkmer H, Wesołowski J (2007) Characterization problems related to the Shepp property. Commun Stat Theory Methods 36(6):1049-1057

Jorgensen B (1982) Statistical properties of the generalized inverse Gaussian distribution. In: Lecture notes in statistics, vol 9. Springer, New York

Letac G, Seshadri V (1985) On Khatri's characterization of the inverse-Gaussian distribution. Can J Stat 13:249-252

Seshadri V (1983) The inverse Gaussian distribution: some properties and characterizations. Can J Stat 11:131-136

Seshadri V (1993) The inverse Gaussian distribution—a case study in exponential families. Oxford University Press, Oxford

Seshadri V, Wesołowski J (2008) More on connections between Wishart and matrix GIG distributions. Metrika 68:219-232

Shepp L (1964) Normal functions of normal random variables. SIAM Rev 6:459-460 